\title{
CURRENT HELICITY OF ACTIVE REGIONS AS A TRACER OF LARGE-SCALE SOLAR MAGNETIC HELICITY
}

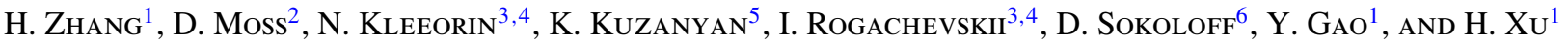 \\ ${ }^{1}$ National Astronomical Observatories, Chinese Academy of Sciences, Beijing 100012, China \\ 2 School of Mathematics, University of Manchester, Manchester M13 9PL, UK \\ ${ }^{3}$ Department of Mechanical Engineering, Ben-Gurion University of Negev, POB 653, 84105 Beer-Sheva, Israel \\ ${ }^{4}$ NORDITA, AlbaNova University Center, Roslagstullsbacken 23, SE-10691 Stockholm, Sweden \\ ${ }^{5}$ IZMIRAN, Troitsk, Moscow Region 142190, Russia \\ ${ }^{6}$ Department of Physics, Moscow State University, Moscow 119992, Russia \\ Received 2011 October 18; accepted 2012 March 17; published 2012 May 3
}

\begin{abstract}
We demonstrate that the current helicity observed in solar active regions traces the magnetic helicity of the large-scale dynamo generated field. We use an advanced two-dimensional mean-field dynamo model with dynamo saturation based on the evolution of the magnetic helicity and algebraic quenching. For comparison, we also studied a more basic two-dimensional mean-field dynamo model with simple algebraic alpha-quenching only. Using these numerical models we obtained butterfly diagrams both for the small-scale current helicity and also for the large-scale magnetic helicity, and compared them with the butterfly diagram for the current helicity in active regions obtained from observations. This comparison shows that the current helicity of active regions, as estimated by $-\mathbf{A} \cdot \mathbf{B}$ evaluated at the depth from which the active region arises, resembles the observational data much better than the small-scale current helicity calculated directly from the helicity evolution equation. Here $\mathbf{B}$ and $\mathbf{A}$ are, respectively, the dynamo generated mean magnetic field and its vector potential. A theoretical interpretation of these results is given.
\end{abstract}

Key words: magnetohydrodynamics (MHD) - Sun: activity - Sun: dynamo - Sun: interior - Sun: magnetic topology - sunspots

Online-only material: color figures

\section{INTRODUCTION}

The solar activity cycle is believed to be a manifestation of dynamo action which somewhere in the solar interior generates waves of quasi-stationary magnetic field propagating from middle latitude toward the solar equator ("dynamo waves"). The traditional explanation of this dynamo action (Parker 1955 ) is based on the joint action of differential rotation and mirror asymmetric convection which results in what has come to be known as the $\alpha$-effect, based on the helicity of the hydrodynamic convective flow (Krause \& Rädler 1980; Moffatt 1978). This explanation is however not the only one discussed in the literature and, for example, meridional circulation is also suggested as an important co-factor of the $\alpha$-effect, see, e.g., Dikpati \& Gilman (2001); Choudhuri et al. (2004).

In turn, traditional dynamo scenarios based on differential rotation and the classical $\alpha$-effect have to include a dynamo saturation mechanism. One of the most popular saturation mechanisms is based on a contribution to the $\alpha$-effect from magnetic fluctuations (Pouquet et al. 1976). A relevant quantification of this effect involves considerations of magnetic helicity evolution, e.g., Kleeorin et al. (1995, 2003). A key role in the evolution of magnetic helicity is played by magnetic helicity fluxes (Kleeorin et al. 2000; Blackman \& Field 2000a; Brandenburg \& Subramanian 2005). Of course, this scenario is not the only one that has been suggested: for example, Blackman \& Field (2000b), Blackman \& Brandenburg (2003), and Brandenburg (2007) consider coronal-mass ejections as an important part of nonlinear suppression of the dynamo, and Mitra et al. (2011) discuss the effects of losses via the solar wind. Choudhuri et al. (2004) believe that the current helicity in solar active regions is substantially modified when magnetic tubes rise up to the solar surface.
A natural way to resolve such controversies is to determine relevant quantities such as the $\alpha$-effect through observations, this providing a check on the various scenarios. Such an option is now becoming realistic, starting from the 1990s when the first attempts to observe current helicity in solar active regions were undertaken (Seehafer 1990; Pevtsov et al. 1994; Bao \& Zhang 1998; Hagino \& Sakurai 2004).

Twenty years of continuous efforts by several observational groups, with the main contribution coming from the Huairou Solar Station of China, has resulted (Zhang et al. 2010) in reconstruction of the current helicity time-latitude (butterfly) diagram for one full solar magnetic cycle (1988-2005). From this butterfly diagram it is apparent that the current helicity is involved in the solar activity cycle and follows a polarity law comparable with the Hale polarity law for sunspots-but rather more complicated. In other words, dynamo generated magnetic field is indeed mirror asymmetric and this mirror asymmetry is involved in the solar activity cycle and can be used to understand its nature (Kleeorin et al. 2003; Zhang et al. 2006).

What, however, needs clarification is which dynamo governing parameter is traced by such a surface proxy as a measure of current helicity in solar active regions. A naive idea here is to identify this part of the surface current helicity with the current and magnetic helicities of the dynamo generated small-scale magnetic fields deep inside the Sun (say, in the solar overshoot layer), which suppress dynamo action. To start with something definite, this naive interpretation was applied by Kleeorin et al. (2003) and Zhang et al. (2006).

Recent progress in observation which has resulted in butterfly diagrams for the current helicity in active regions (Zhang et al. 2010) makes it possible to go further with this topic. The aim of this paper is to argue that the current helicity in solar active regions directly reflects the magnetic helicity of the 
large-scale dynamo generated field. We organize our arguments as follows.

Based on the observed butterfly diagram for the current helicity, we here confront the naive interpretation with the available observations. We examine a mean-field dynamo model with dynamo suppression based on the magnetic helicity balance, obtain the corresponding current helicity butterfly diagrams, and also that of the large-scale magnetic helicity, and compare them with those observed. This comparison shows that the evolution of the large-scale magnetic helicity resembles the observational data much more closely than that of the current helicity of the small-scale fields. In order to demonstrate the robustness of this result, we also consider a more primitive dynamo model, with a simple algebraic $\alpha$-quenching. From this model we calculate the large-scale magnetic helicity and compare it with the observational butterfly diagram. We find that this fits observations more or less as well as that from a dynamo model based on helicity conservation. We conclude that the major part of the observed current helicity in active regions is produced in the rise of magnetic loops to the solar surface.

\section{THE ROLE OF HELICITIES IN MAGNETIC FIELD EVOLUTION}

In the evolution of the magnetic field different helicities play different roles. Considering the small-scale velocity and magnetic fluctuations, $\mathbf{u}$ and $\mathbf{b}$, respectively, there are three helicities: (1) the kinetic helicity $H_{u}=\langle\mathbf{u} \cdot \operatorname{curl} \mathbf{u}\rangle$ that determines the kinetic $\alpha$ effect; (2) the current helicity $H_{c}=\langle\mathbf{b} \cdot \operatorname{curl} \mathbf{b}\rangle$ that determines the magnetic part of the $\alpha$ effect; and (3) the magnetic helicity $H_{m}=\langle\mathbf{a} \cdot \mathbf{b}\rangle$, where $\mathbf{b}=$ curl $\mathbf{a}$. We use the angled brackets $\langle\cdots\rangle$ to denote spatial integrals over all relevant turbulent fluid. These integrated helicities are used in the sense of average helicity densities.

The total magnetic helicity, the sum $H_{M}+H_{m}$ of the magnetic helicities of the large- and small-scale fields, $H_{M}=\mathbf{A} \cdot \mathbf{B}$ and $H_{m}$, respectively, is conserved for very large magnetic Reynolds numbers. Here, $\mathbf{B}=\operatorname{curl} \mathbf{A}$ is the large-scale magnetic field. Note that, on the contrary, the current helicity $H_{c}$ is not conserved. On the other hand, the kinetic helicity $H_{u}$ is conserved only for very large fluid Reynolds numbers when the large-scale magnetic field $\mathbf{B}$ vanishes. The characteristic time for the decay of kinetic helicity is of the order of the turnover time $\tau=\ell / u$ of turbulent eddies in the energycontaining scale, $\ell$, of turbulence, while the characteristic time of the small magnetic helicity decay is of the order of $T_{m}=\tau \operatorname{Rm}$ (Moffatt 1978; Zeldovich et al. 1983; Brandenburg \& Subramanian 2005), where $\mathrm{Rm}=\ell u / \eta_{0}$ is the magnetic Reynolds number, $u$ is the characteristic turbulent velocity, and $\eta_{0}$ is the magnetic diffusivity due to electrical conductivity of the fluid. The small-scale current helicity, $H_{c}$, is not an integral of motion and the characteristic time of $H_{c}$ varies from a short timescale, $\tau$, to much larger timescales. On the other hand, the characteristic decay times of the current helicity of large-scale field, $H_{C}=\mathbf{B} \cdot \operatorname{curl} \mathbf{B}$, and of the large-scale magnetic helicity, $H_{M}$, are of the order of the turbulent diffusion time. For weakly inhomogeneous turbulence the small-scale current helicity, $H_{c}$, is proportional to the small-scale magnetic helicity, $H_{m}$.

As the dynamo amplifies the large-scale magnetic field, the large-scale magnetic helicity $H_{M}=\mathbf{A} \cdot \mathbf{B}$ grows in time (but not monotonically in a cyclic system). The evolution of the large- scale magnetic helicity $H_{M}$ is determined by

$$
\frac{\partial H_{M}}{\partial t}+\nabla \cdot \mathbf{F}_{M}=2 \mathcal{E} \cdot \mathbf{B}-2 \eta H_{C}
$$

(Kleeorin et al. 1995; Blackman \& Field 2000a; Brandenburg $\&$ Subramanian 2005), where $\mathcal{E}=\langle\mathbf{u} \times \mathbf{b}\rangle$ is the mean electromotive force that determines generation and dissipation of the large-scale magnetic field, $2 \mathcal{E} \cdot \mathbf{B}$ is the source of the large-scale magnetic helicity due to the dynamo generated large-scale magnetic field, and $\mathbf{F}_{M}$ is the flux of large-scale magnetic helicity that determines its transport. Since the total magnetic helicity over all scales, $H_{M}+H_{m}$ integrated over the turbulent fluid, is conserved for very small magnetic diffusivity, the small-scale magnetic helicity changes during the dynamo process, and its evolution is determined by the dynamic equation

$$
\frac{\partial H_{m}}{\partial t}+\nabla \cdot \mathbf{F}=-2 \mathcal{E} \cdot \mathbf{B}-2 \eta H_{c}
$$

(Kleeorin et al. 1995; Blackman \& Field 2000a; Brandenburg $\&$ Subramanian 2005), where $-2 \mathcal{E} \cdot \mathbf{B}$ is the source of the small-scale magnetic helicity due to the dynamo generated large-scale magnetic field, $\mathbf{F}$ is the flux of small-scale magnetic helicity that determines its transport, and $2 \eta H_{c}=H_{m} / T_{m}$ is the dissipation rate of the small-scale magnetic helicity. It follows from Equations (1) and (2) that the source of the small-scale and the large-scale magnetic helicities is located only in turbulent regions (i.e., in our case, in the solar convective zone). The magnetic part of the $\alpha$ effect is determined by the parameter $\chi^{c}=\tau H_{c} /(12 \pi \rho)$, and for weakly inhomogeneous turbulence $\chi^{c}$ is proportional to the magnetic helicity: $\chi^{c}=H_{m} /\left(18 \pi \eta_{T} \rho\right)$ (Kleeorin \& Rogachevskii 1999; Brandenburg \& Subramanian 2005), where $\rho$ is the density and $\eta_{T}$ is the turbulent magnetic diffusion.

\section{THE OBSERVED CURRENT HELICITY BUTTERFLY DIAGRAMS}

The observed butterfly diagrams of electric current helicity for solar active regions during the last two solar cycles have been presented by Zhang et al. (2010). The general structure can be described as follows. Current helicity is involved in the solar activity cycle and follows a polarity rule comparable to (however more complicated than) the polarity rule for toroidal magnetic field which in turn comes from the Hale polarity rule for sunspot groups. Migration of the helicity pattern is clearly visible and located near the toroidal field pattern. The wings of the helicity butterflies are slightly more inclined to the equator than the magnetic field wings, but the former follow in general the latter. As a quantity quadratic in magnetic field, the current helicity in one hemisphere has the same sign for both cycles, with the opposite sign in the other hemisphere (a kind of unchanging dipolar symmetry).

Figure 1 shows the distribution of the average helical characteristics of the magnetic field in solar active regions in the form of a butterfly diagram (latitude-time) for 1988-2005 (which covers most of the 22 nd and 23 rd solar cycles). These results are inferred from photospheric vector magnetograms recorded at Huairou Solar Observing Station.

This, the longest available systematic data set covering the period of two solar cycles, comprises 6205 vector magnetograms of 984 solar active regions (most of the large solar active regions of both solar cycles). Of these, 431 active regions belong to 


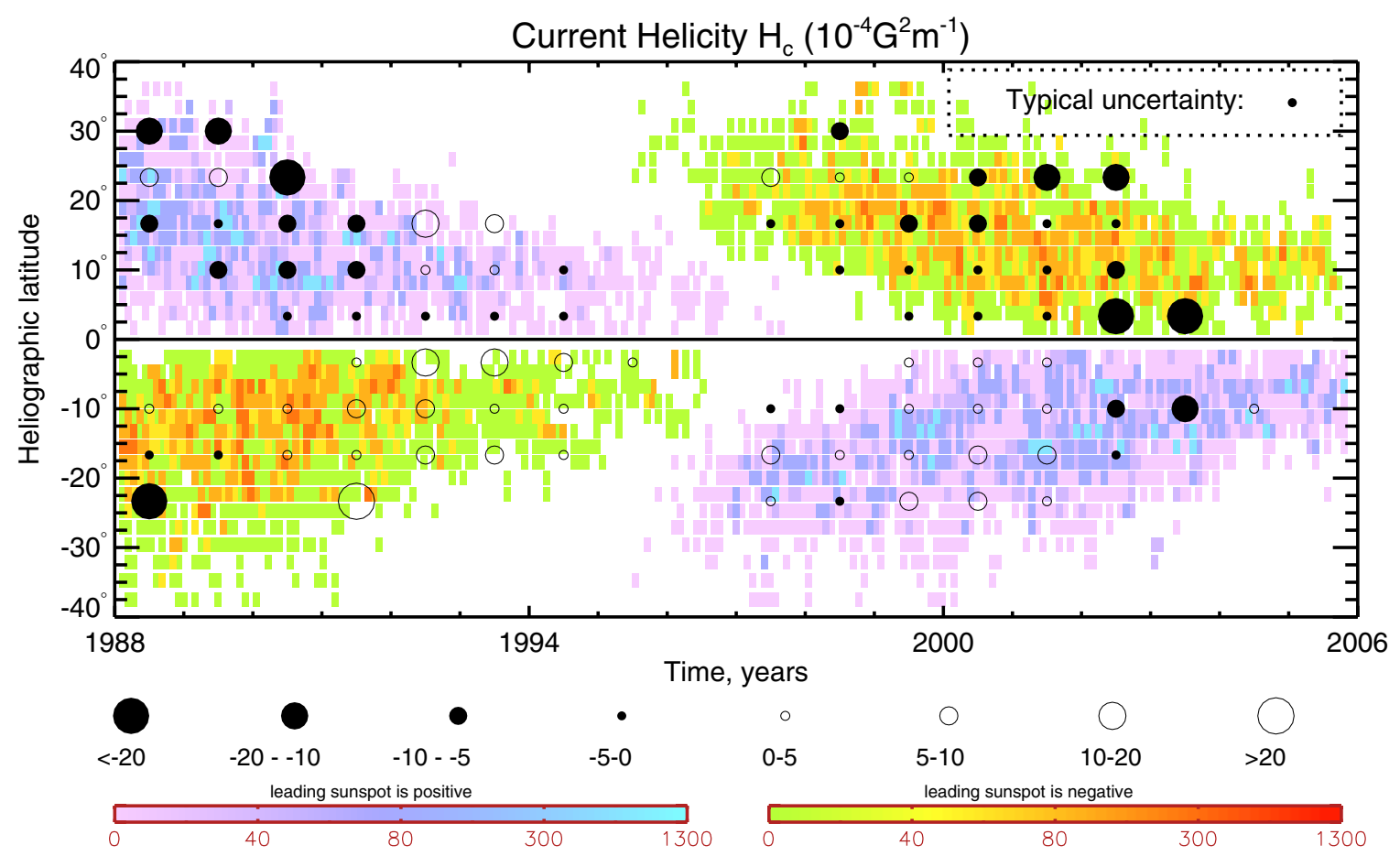

Figure 1. Observed current helicity (white/black circles for positive/negative values) for solar active regions in the 22nd and 23rd solar cycles as averaged over two-year running windows over latitudinal bins of $7^{\circ}$ wide, overlaid with sunspot density (color). The circle in the upper right corner of the panel indicates the typical value of observational uncertainty defined by $95 \%$ confidence intervals scaled to the same units as the circles. The vertical axis gives the latitude in degrees and the horizontal gives the time in years.

(A color version of this figure is available in the online journal.)

the 22 nd solar cycle and 553 to the 23 rd. We have limited the latitudes of active regions to $\pm 40^{\circ}$ and most of them are below $\pm 35^{\circ}$. The helicity values of the active regions have been averaged over latitude by intervals of $7^{\circ}$ in solar latitude, and over overlapping two-year periods of time (i.e., 1988-1989, 1989-1990, .., 2004-2005). By this method of averaging we were able to group sets of at least 30 data points in order to make error estimations (computed as $95 \%$ confidence intervals) reasonably small. In this sampling we find that $66 \%$ (63\%) of active regions have negative (positive) mean current helicity in the northern (southern) hemisphere over the 22nd solar cycle and $58 \%(57 \%)$ in the 23 rd solar cycle.

There are some domains in the diagram where current helicity has the "wrong" sign with respect to the global polarity law. These domains of "wrong" sign are located at the very beginning and the very end of the wings.

Concerning alternative explanations of the observations (Zhang et al. 2010), Mackay \& Van Ballegooijen (2005) and Yeates \& Mackay (2009) describe current helicity in solar active regions in terms of generation of non-potential coronal structures by surface differential rotation. Note that the surface differential rotation cannot generate large-scale and small-scale magnetic helicity. It can only redistribute the existing magnetic helicity, as can any non-uniform large-scale motions. This process is determined by the flux term in the evolutionary equation for the magnetic helicity. The local change of the magnetic helicity inside a given active region by the surface differential rotation plays an important role. In our paper, we study robust global (rather than local) features of the evolution of the magnetic helicity, by averaging over an ensemble of active regions. In this case the global evolution of the magnetic helicity mainly depends on the sources of magnetic helicity inside the solar convective zone.

\section{AN ESTIMATE FOR THE CURRENT HELICITY IN ACTIVE REGIONS}

In this section, we estimate the current helicity in active regions. There is a common belief that active regions are formed due to some nonaxisymmetric instability of $\sim 100 \mathrm{kG}$ magnetic fields in the tachocline (e.g., Gilman \& Dikpati 2000; Cally et al. 2003; Parfrey \& Menou 2007). However, the existence of such strong fields and the role of this mechanism remain questionable (Brandenburg 2005). Another promising mechanism of formation of active regions is related to a negative effective magnetic pressure instability of the largescale dynamo generated magnetic field. This instability was predicted theoretically (Kleeorin et al. 1996; Rogachevskii \& Kleeorin 2007) and detected recently in direct numerical simulations (Brandenburg et al. 2011). The instability is caused by the suppression of turbulent hydromagnetic pressure by the mean magnetic field. At large Reynolds numbers and for sub-equipartition magnetic fields, the resulting negative turbulent contribution can become so large that the effective mean magnetic pressure (the sum of turbulent and non-turbulent contributions) appears negative (Brandenburg et al. 2010, 2011). In a stratified turbulent convection, this results in the excitation of a large-scale instability that results in the formation of largescale inhomogeneous magnetic structures. This mechanism is consistent with the suggestion that active regions are formed near the surface of convective zone (Kosovichev 2010).

The spatial scale of an active region is much smaller than the solar radius, but much larger then the maximum scale of solar turbulent granulation. To estimate the current helicity in an active region, we have to relate the large-scale magnetic field $\mathbf{B}$ and its magnetic potential $\mathbf{A}$ inside the convective zone as well as the corresponding small-scale quantities inside the convective 
zone (which determine the small-scale magnetic fluctuations), with the surface magnetic field $\mathbf{B}^{\text {ar }}$ and its magnetic potential $\mathbf{A}^{\text {ar }}$ inside active regions, which are the quantities available to observations.

We base our estimate on the following idea. Consider a newborn magnetic tube at the layer in convective zone from which the active region is developing. We assume that the rise of magnetic tubes is a fast adiabatic process. Let us also assume that the mean magnetic field and the total magnetic helicity vanish at the initial instant, and take into account the magnetic helicity conservation law (as solar plasma is highly conductive, and so we consider the magnetic helicity conservation law to hold at all scales, including that of the whole Sun). If this tube rises rapidly to the surface to produce an active region, the total magnetic helicity in the tube is conserved because the process is rapid. Rising large-scale magnetic field and magnetic potential give the corresponding quantities for active regions, which may thus differ substantially from the corresponding quantities in the surrounding medium. Because the initial total magnetic helicity of the tube, which was almost nonmagnetized, was negligible, the magnetic helicity conservation law reads

$$
\left\langle\mathbf{A}^{\mathrm{ar}} \cdot \mathbf{B}^{\mathrm{ar}}\right\rangle \approx-\boldsymbol{A} \cdot \boldsymbol{B},
$$

where the angular brackets denote averaging over the surface occupied by the active region.

Now we relate the mean current helicity $\left\langle\mathbf{B}^{\text {ar }} \cdot \operatorname{curl} \mathbf{B}^{\text {ar }}\right\rangle$ with the magnetic helicity $\left\langle\mathbf{A}^{\mathrm{ar}} \cdot \mathbf{B}^{\mathrm{ar}}\right\rangle$. We rewrite it from the first principles with the use of permutation tensors as

$$
\left\langle\mathbf{B}^{\mathrm{ar}} \cdot \operatorname{curl} \mathbf{B}^{\mathrm{ar}}\right\rangle \approx \frac{1}{L_{\mathrm{ar}}^{2}}\left\langle\mathbf{A}^{\mathrm{ar}} \cdot \mathbf{B}^{\mathrm{ar}}\right\rangle+O\left(\frac{L_{\mathrm{ar}}^{2}}{R_{\odot}^{2}}\right),
$$

(see Appendix A), where $R_{\odot}$ is the solar radius and $L_{\mathrm{ar}}$ is the spatial scale of an active region. Equations (3) and (4) yield

$$
\left\langle\mathbf{B}^{\mathrm{ar}} \cdot \operatorname{curl} \mathbf{B}^{\mathrm{ar}}\right\rangle \approx-\frac{1}{L_{\mathrm{ar}}^{2}} \boldsymbol{A} \cdot \boldsymbol{B} .
$$

Therefore, the observed current helicity in active regions is expected to be a proxy for $-\mathbf{A} \cdot \mathbf{B}$. This idea will be checked using mean-field dynamo numerical modeling and comparison of the numerical results with the observed current helicity in active regions.

\section{DYNAMO MODELS}

Our approach to compare the dynamo models with observations is as follows. We consider two types of dynamo models. Both types of models are two-dimensional mean-field models with an axisymmetric magnetic field which depends on radius $r$ and polar angle $\theta$. The third (azimuthal) coordinate is $\phi$ and $\partial / \partial \phi=0$. The dynamo action is based on differential rotation, with a rotation curve which resembles that of the solar convection zone, as known from helioseismological observations, and there is a conventional $\alpha$-effect.

The first type of model assumes a very naive algebraic $\alpha$ quenching. Then we suppose that the total magnetic helicity is locally vanishing, so the magnetic helicity of the large-scale magnetic field produced in the course of mean-field dynamo action has to be compensated by small-scale magnetic helicity. (Thus, we are assuming that at an initial instant the medium is non-magnetic, so that helicity conservation means that the sum of large- and small-scale helicities remain zero.) We assume also that there is a separation of scales so that characteristic turbulence scales are much smaller than the characteristic spatial scales of mean magnetic field variations. This allows a link between current and magnetic helicities (Kleeorin \& Rogachevskii 1999) to be made. This concept underlies the observational procedure for determining the current helicity of active regions, and for calculating the current helicity from the magnetic helicity of the small-scale fields. Based on the same concept we estimate the large-scale magnetic helicity as $B_{\phi} A_{\phi}$, where $A(r, \theta) \hat{\phi}$ is the magnetic potential for the poloidal field. As a result we obtain (for a given radius $r$ ) a theoretical model for the current helicity as a function of $t$ and $\theta$ which we overlay on the butterfly diagram for $B_{\phi}$. We compare the result with the current helicity butterfly diagram known from observations and obtained using similar underlying concepts.

A further point is that the primitive model allows a simplification to the level of the one-dimensional Parker migratory dynamo, and this opportunity has been investigated in this respect by Xu et al. (2009). We will use the results of that work for reference and comparison.

We do not consider this primitive scheme as realistic. We are sure that any more or less realistic scenario for solar dynamo suppression will have to be much more sophisticated. On the other hand, we can see whether this primitive model produces a helicity butterfly diagram that is quite similar to that observed. The only shortcoming of the model is that the maximal current helicity occurs later then the maximum $B_{\phi}$, while it is observed to come up earlier. If magnetic helicity conservation determines the nonlinear dynamo suppression, we expect that a careful reproduction of this balance, including helicity fluxes and the link between magnetic helicity and $\alpha$-effect, will result in an even better theoretical butterfly diagram, and possibly improve the phase relations between helicity and toroidal magnetic field.

As a specific example of the second-type model that takes into account the influence of magnetic helicity balance on dynamo action we use the dynamo model described by Zhang et al. (2006). Whereas simple $\alpha$-quenching provides a quite robust suppression of a spherical dynamo and give (more or less) steady nonlinear magnetic field oscillations for a very wide range of parameters, in contrast it is far from clear a priori that a dynamo suppression based on magnetic helicity conservation is effective enough to suppress magnetic field growth and result in steady oscillations. In fact it works more or less satisfactorily only in a quite narrow parameter range (Kleeorin et al. 2003; Zhang et al. 2006), which appears inadequate to fit observations.

We note two crucial points here. First of all, both types of models ignore any direct action of magnetic force on the rotation law. In the more primitive models, there is a crude parameterization of feedback onto the (purely hydrodynamic) alpha effect (see Equation (6)) below. The latter more sophisticated model describes the back reaction of the generated magnetic field on the dynamo process in terms of the magnetic contribution of the current helicity onto the magnetic part of $\alpha$-effect. On the other hand, the feedback of the generated large-scale magnetic field on turbulent convection is described in our model by the algebraic quenching of $\alpha$-effect, pumping velocities, and turbulent magnetic diffusion.

We assume that helicity conservation is not the only mechanism of dynamo suppression. The fact that we see a manifestation of helicity on the solar surface tells us that the buoyancy indeed plays some role, and we add it to the model. We stress that the buoyancy which we include in the model transports current 
helicity and magnetic helicity as well as large-scale magnetic field.

Below we discuss the detailed dynamo models. We use spherical coordinates $r, \theta, \phi$ and describe an axisymmetric magnetic field by the azimuthal component of magnetic field $B$, and the component $A$ of the magnetic potential corresponding to the poloidal field.

We measure length in units of the solar radius $R_{\odot}$, and time in units of a diffusion time based on the solar radius and the reference turbulent magnetic diffusivity $\eta_{T 0}$. The magnetic field is measured in units of the equipartition field $B_{\mathrm{eq}}=u_{*}\left(4 \pi \rho_{*}\right)^{1 / 2}$, the vector potential of the poloidal field $A$ in units of $R_{\odot} B_{\text {eq }}$, the density $\rho$ is normalized with respect to its value $\rho_{*}$ at the bottom of the convective zone, and the basic scales of the turbulent motions $\ell$ and turbulent velocity $u$ at the scale $\ell$ are measured in units of their maximum values through the convective zone. The $\alpha$-effect is measured in terms of $\alpha_{0}$, defined below, and angular velocity in units of the maximum surface value, $\Omega_{0}$.

\subsection{The Primitive, Alpha-quenched Model}

In the primitive dynamo model the $\alpha$-effect is given by

$$
\alpha=\alpha^{v}=\chi^{v} \Phi_{v},
$$

where $\chi^{v}$ is proportional to the hydrodynamic helicity, $H_{u}$, multiplied by the turbulent correlation time $\tau$, and $\Phi_{v}=$ $\left(1+B^{2}\right)^{-1}$ is the model for the $\alpha$-quenching nonlinearity. For convenience, we use for most of these computations the code of Moss \& Brooke (2000)—see also Moss et al. (2011). This code has the possibility of a modest reduction in the diffusivity, to $\eta_{\min }$, in the innermost part of the computational shell ("tachocline"), below fractional radius 0.7 . We define $\eta_{\mathrm{r}}=\eta_{\min } / \eta_{T 0}$. We also used this primitive formulation of alpha-quenching in the (otherwise very similar) model used in Section 5.2 when producing Figure 4. In the latter case, the diffusivity is everywhere uniform.

At the surface $r=1$ the field is matched to a vacuum external field, and "overshoot" boundary conditions are used at the lower boundary.

\subsection{The Model Based on Helicity Balance}

Here we use the code described in Zhang et al. (2006), with two new features: we allow the possibility of meridional circulation and/or vertical motions attributed to magnetic buoyancy. The dynamo equations for $\tilde{A}=r \sin \theta A$ and $\tilde{B}=r \sin \theta B$ read

$$
\begin{gathered}
\frac{\partial \tilde{A}}{\partial t}+\frac{\left(V_{\theta}^{A}+V_{\theta}^{M}\right)}{r} \frac{\partial \tilde{A}}{\partial \theta}+V_{r} \frac{\partial \tilde{A}}{\partial r}=C_{\alpha} \alpha \tilde{B} \\
+\eta_{A}\left[\frac{\partial^{2} \tilde{A}}{\partial r^{2}}+\frac{\sin \theta}{r^{2}} \frac{\partial}{\partial \theta}\left(\frac{1}{\sin \theta} \frac{\partial \tilde{A}}{\partial \theta}\right)\right] \\
\frac{\partial \tilde{B}}{\partial t}+\frac{\sin \theta}{r} \frac{\partial}{\partial \theta}\left[\frac{\left(V_{\theta}^{B}+V_{\theta}^{M}\right) \tilde{B}}{\sin \theta}\right]+\frac{\partial\left[V_{r} \tilde{B}\right]}{\partial r} \\
=C_{\omega} \sin \theta\left[G_{r} \frac{\partial}{\partial \theta}-G_{\theta} \frac{\partial}{\partial r}\right] \tilde{A}+\frac{\sin \theta}{r^{2}} \frac{\partial}{\partial \theta}\left[\frac{\eta_{B}}{\sin \theta} \frac{\partial \tilde{B}}{\partial \theta}\right] \\
+\frac{\partial}{\partial r}\left[\eta_{B} \frac{\partial \tilde{B}}{\partial r}\right],
\end{gathered}
$$

where $V_{r}=V_{r}^{A}+V_{r}^{M}+V_{B}, V^{A}(B)$ and $V^{B}(B)$ are the nonlinear drift velocities of poloidal and toroidal mean magnetic fields, $V^{M}$ is the meridional circulation velocity, $\mathbf{V}_{\mathrm{B}}$ is the vertical buoyancy velocity, $\eta_{A}(B)$ and $\eta_{B}(B)$ are the nonlinear turbulent magnetic diffusion coefficients for the mean poloidal and toroidal magnetic fields, and the non-dimensional dynamo parameters are $C_{\alpha}=\alpha_{0} R_{\odot} / \eta_{T 0}, C_{\omega}=\Omega_{0} R_{\odot}^{2} / \eta_{T 0}$. The nonlinear turbulent magnetic diffusion coefficients and the nonlinear drift velocities are given in Appendix B. The nondimensional gradients of differential rotation are

$$
G_{r}=\frac{\partial \Omega}{\partial r}, \quad G_{\theta}=\frac{\partial \Omega}{\partial \theta} .
$$

In this dynamo model with magnetic helicity evolution the total $\alpha$-effect is given by

$$
\alpha=\alpha^{v}+\alpha^{m}=\chi^{v} \Phi_{v}+\frac{\Phi_{m}}{\rho(z)} \chi^{c},
$$

with $\alpha^{v}=\alpha_{0} \sin ^{2} \theta \cos \theta \Phi_{v}$. The magnetic part of the $\alpha$-effect is based on the idea of magnetic helicity conservation and the link between current and magnetic helicities. Here $\chi^{v}$ and $\chi^{c}$ are proportional to the hydrodynamic and current helicities multiplied by the turbulent correlation time, and $\Phi_{v}$ and $\Phi_{m}$ are quenching functions. The analytical form of the quenching functions $\Phi_{v}(B)$ and $\Phi_{m}(B)$ is given in Appendix B. The density profile is chosen in the form:

$$
\rho(z)=\exp [-a \tan (0.45 \pi z)]
$$

where $z=1-\mu(1-r)$ and $\mu=\left(1-R_{0} / R_{\odot}\right)^{-1}$. Here, $a \approx 0.3$ corresponds to a tenfold change of the density in the solar convective zone, $a \approx 1$ by a factor of about $10^{3}$.

The equation for $\tilde{\chi}^{c}=r^{2} \sin ^{2} \theta \chi^{c}$ is

$$
\begin{aligned}
\frac{\partial \tilde{\chi}^{c}}{\partial t}+\frac{\tilde{\chi}^{c}}{T}= & \left(\frac{2 R_{\odot}}{\ell}\right)^{2}\left\{\frac { 1 } { C _ { \alpha } } \left[\frac{\eta_{B}}{r^{2}} \frac{\partial \tilde{A}}{\partial \theta} \frac{\partial \tilde{B}}{\partial \theta}+\eta_{B} \frac{\partial \tilde{A}}{\partial r} \frac{\partial \tilde{B}}{\partial r}\right.\right. \\
& -\eta_{A} \tilde{B} \frac{\sin \theta}{r^{2}} \frac{\partial}{\partial \theta}\left(\frac{1}{\sin \theta} \frac{\partial \tilde{A}}{\partial \theta}\right)-\eta_{A} \tilde{B} \frac{\partial^{2} \tilde{A}}{\partial r^{2}} \\
& \left.\left.+\left(V_{r}^{A}-V_{r}^{B}\right) \tilde{B} \frac{\partial \tilde{A}}{\partial r}+\left(V_{\theta}^{A}-V_{\theta}^{B}\right) \frac{\tilde{B}}{r} \frac{\partial \tilde{A}}{\partial \theta}\right]-\alpha \tilde{B}^{2}\right\} \\
& -\frac{\partial\left[\tilde{\mathcal{F}}_{r}+\left(V_{B}+V_{r}^{M}\right) \tilde{\chi}^{c}\right]}{\partial r} \\
& -\frac{\sin \theta}{r} \frac{\partial}{\partial \theta}\left[\frac{\tilde{\mathcal{F}}_{\theta}+V_{\theta}^{M} \tilde{\chi}^{c}}{\sin \theta}\right],
\end{aligned}
$$

where $\tilde{\mathcal{F}}=r^{2} \sin ^{2} \theta \mathcal{F}, \mathcal{F}=\mathbf{F} /\left(18 \pi \eta_{T} \rho\right)$ is related to the flux $\mathbf{F}$ of the small-scale magnetic helicity and given in Appendix B, $R_{\odot} / \ell$ is the ratio of the solar radius to the basic scale of solar convection, $T=(1 / 3) \operatorname{Rm}\left(\ell / R_{\odot}\right)^{2}$ is the dimensionless relaxation time of the magnetic helicity, $\mathrm{Rm}=\ell u / \eta_{0}$ is the magnetic Reynolds number, with $\eta_{0}$ the "basic" magnetic diffusion due to the electrical conductivity of the fluid.

The meridional circulation (single cell in each hemisphere, poleward at surface) is determined by

$$
\begin{gathered}
V_{\theta}^{M}=-\frac{1}{\sin \theta r \rho(r)} \frac{\partial[r \Psi(r, \theta)]}{\partial r}, \\
V_{r}^{M}=\frac{1}{\sin \theta r \rho(r)} \frac{\partial \Psi(r, \theta)}{\partial \theta},
\end{gathered}
$$



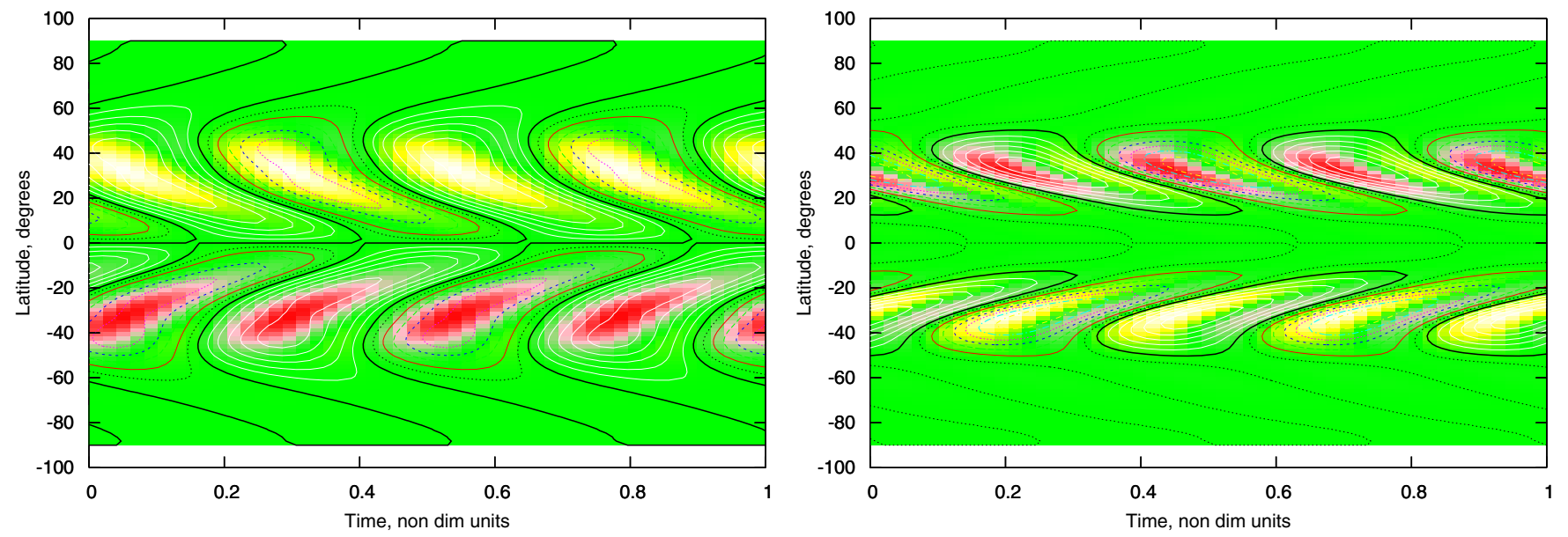

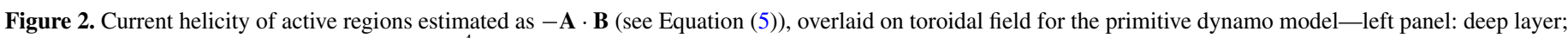

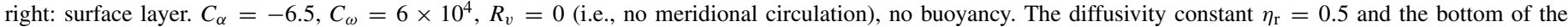
computational region is at $r_{0}=0.64$. The color palette is hereafter chosen as follows: yellow is positive, red is negative, and green is zero.

(A color version of this figure is available in the online journal.)

where $\Psi(r, \theta)=R_{v} \sin ^{2} \theta \cos \theta f(r) \rho, f(r)=2\left(r-r_{b}\right)^{2}(r-$ 1)/(1-r $\left.r_{b}\right)^{2}, r_{b}$ is the base of the computational shell. This is normalized so that the max of $V_{\theta}^{M}$ at the surface is unity. We introduce a coefficient $R_{v}=R_{\odot} U_{0} / \eta_{T 0}$, where $U_{0}$ the maximum surface speed.

Buoyancy is implemented by the introduction of a purely vertical velocity $\mathbf{V}_{\mathrm{B}}=\gamma B_{\phi}^{2} \hat{\mathbf{r}}$ in Equations (7), (8), and (11), where $\gamma>0$ (Moss et al. 1999). We justify the apparent nonconservation of mass by adopting the argument of K.-H. Rädler, presented as a private communication in D. Moss et al. (1990), that the return velocity will be in the form of a more-or-less uniform "rain." In some ways the process represents pumping by a "fountain flow." As a result the regular velocity $\mathbf{V}_{B}$ appears in the governing equations for the large-scale magnetic field and magnetic and current helicities but not the equation for density. From the viewpoint of probability theory, in the first case $\mathbf{V}_{B}$ is a mean quantity taken under the condition that an elementary volume is magnetized so it does not vanish, while in the second case this mean is taken without any condition and vanishes. In our opinion, this idea can also be constructive for other problems with magnetic helicity advective fluxes, e.g., Shukurov at al. (2006).

At the surface of the Sun, $r=1$, we use vacuum boundary conditions on the field, i.e., $B=0$ and the poloidal field fits smoothly onto a potential external field. At the lower boundary (the bottom of the solar convective zone), $r=r_{0}=0.64, B=$ $B_{r}=0$. At both $r=r_{0}$ and $r=1$, we set $\partial \chi^{c} / \partial r=0$, where $\chi^{c}$ is proportional to the current helicity (see Equation (9)).

\section{SIMULATED BUTTERFLY DIAGRAMS FOR CURRENT HELICITY}

We performed an extensive numerical investigation of the models in a parametric range which is considered to be adequate for solar dynamos. We estimate the values of the governing parameters for different depths of the convective zone, using models of the solar convective zone, e.g., Baker \& Temesvary (1966) and Spruit (1974) — more modern treatments make little difference to these estimates. In the upper part of the convective zone, say at depth (measured from the top) $h_{*}=2 \times 10^{7} \mathrm{~cm}$, the parameters are $\mathrm{Rm}=10^{5}, u=9.4 \times 10^{4} \mathrm{~cm} \mathrm{~s}^{-1}, \ell=$ $2.6 \times 10^{7} \mathrm{~cm}, \rho=4.5 \times 10^{-7} \mathrm{~g} \mathrm{~cm}^{-3}$, the turbulent diffusivity
$\eta_{T}=0.8 \times 10^{12} \mathrm{~cm}^{2} \mathrm{~s}^{-1}$; the equipartition mean magnetic field is $B_{\text {eq }}=220 \mathrm{G}$ and $T=5 \times 10^{-3}$. At depth $h_{*}=10^{9} \mathrm{~cm}$, these values are $\mathrm{Rm}=3 \times 10^{7}, u=10^{4} \mathrm{~cm} \mathrm{~s}^{-1}, \ell=2.8 \times 10^{8} \mathrm{~cm}$, $\rho=5 \times 10^{-4} \mathrm{~g} \mathrm{~cm}^{-3}, \eta_{T}=0.9 \times 10^{12} \mathrm{~cm}^{2} \mathrm{~s}^{-1}$; the equipartition mean magnetic field is $B_{\mathrm{eq}}=800 \mathrm{G}$ and $T \sim 150$. At the bottom of the convective zone, say at depth $h_{*}=2 \times 10^{10} \mathrm{~cm}$, $\mathrm{Rm}=2 \times 10^{9}, u=2 \times 10^{3} \mathrm{~cm} \mathrm{~s}^{-1}, \ell=8 \times 10^{9} \mathrm{~cm}$, $\rho=2 \times 10^{-1} \mathrm{~g} \mathrm{~cm}^{-3}, \eta_{T}=5.3 \times 10^{12} \mathrm{~cm}^{2} \mathrm{~s}^{-1}$. Here the equipartition means magnetic field $B_{\mathrm{eq}}=3000 \mathrm{G}$ and $T \approx 10^{7}$. If we average the parameter $T$ over the depth of the convective zone, we obtain $T \approx 5$, see Kleeorin et al. (2003).

We start with the results for the primitive model. Figure 2 presents the current helicity butterfly diagrams overlaid on those for the toroidal field. We estimate this quantity based on the idea that the observed current helicity in active regions is expected to trace $-\mathbf{A} \cdot \mathbf{B}$, and so we plot in this section $-\mathbf{A} \cdot \mathbf{B}$.

We see that the plots successfully represent main feature of the observed helicity patterns. The pattern presented in Figure 2 is quite typical for the model. Of course, one can choose a set of dynamo governing parameters which is less similar to the observations. For example one can concentrate magnetic fields in the deep layer of convective zone (say, in the overshoot layer) by choosing a reduction $\eta_{\mathrm{r}}=0.1$ in the nominal "overshoot layer," instead of $\eta_{\mathrm{r}}=0.5$ (our standard case), as used in Figure 2. This tends to make the helicity wave in the overshoot layer look more like a standing wave, but keeps the main features of surface diagram (Figure 3 ). The highly anharmonic standing patterns of the butterfly diagram that were discussed as a possible option for some stars, see Baliunas et al. (2006), look however irrelevant for the solar case.

Of course, the helicity pattern in the butterfly diagram obtained in the models for particular choices of dynamo governing parameters can be slightly different from the observed helicity patterns. Xu et al. (2009) demonstrated that meridional circulation can be used to make the simulated pattern resemble more closely that observed.

We produced the same type of plots for models based on helicity conservation Figure 4 . We also present in Figure 5 the small-scale current helicity $\chi_{c}$. Here the migration diagrams are presented for the middle radius of the dynamo region: nearer the surface $\chi_{c}$ displays only relatively weak vacillatory behavior. We see that the small-scale current helicity is strongly 

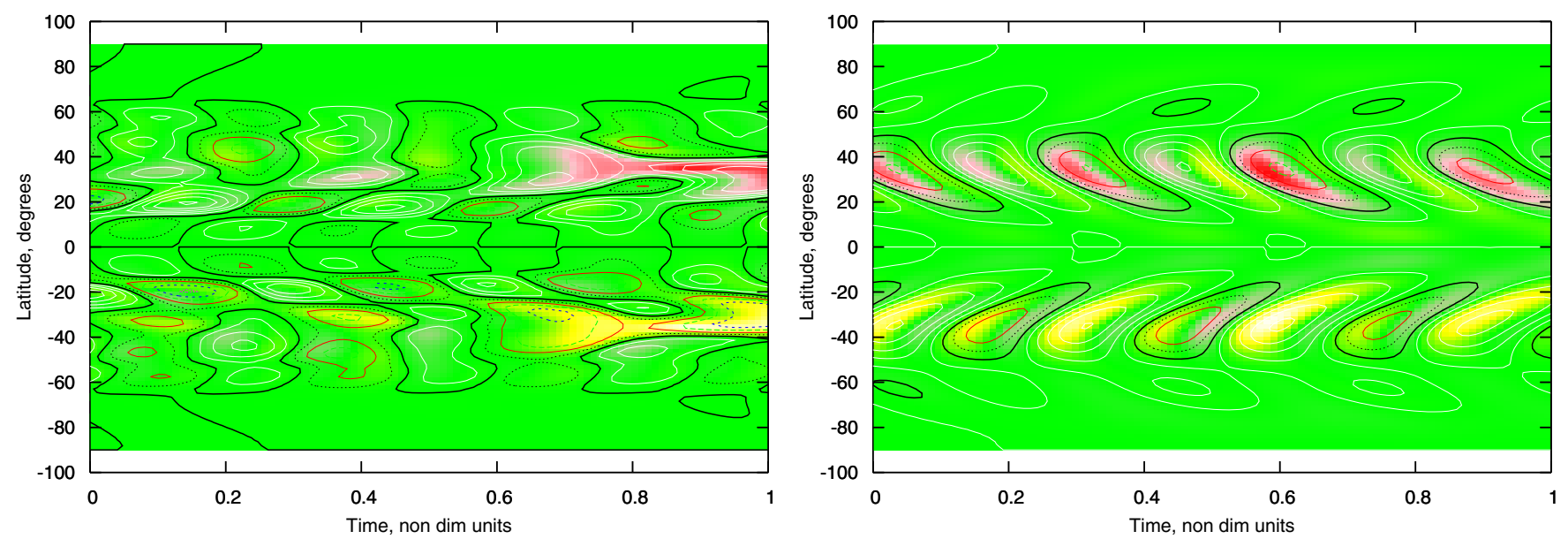

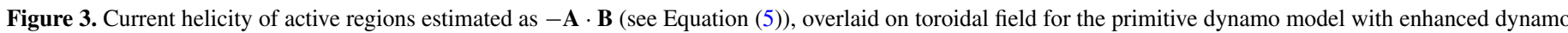

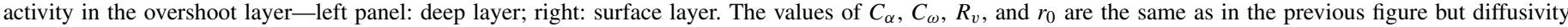
contrast $\eta_{\mathrm{r}}=0.1$.

(A color version of this figure is available in the online journal.)
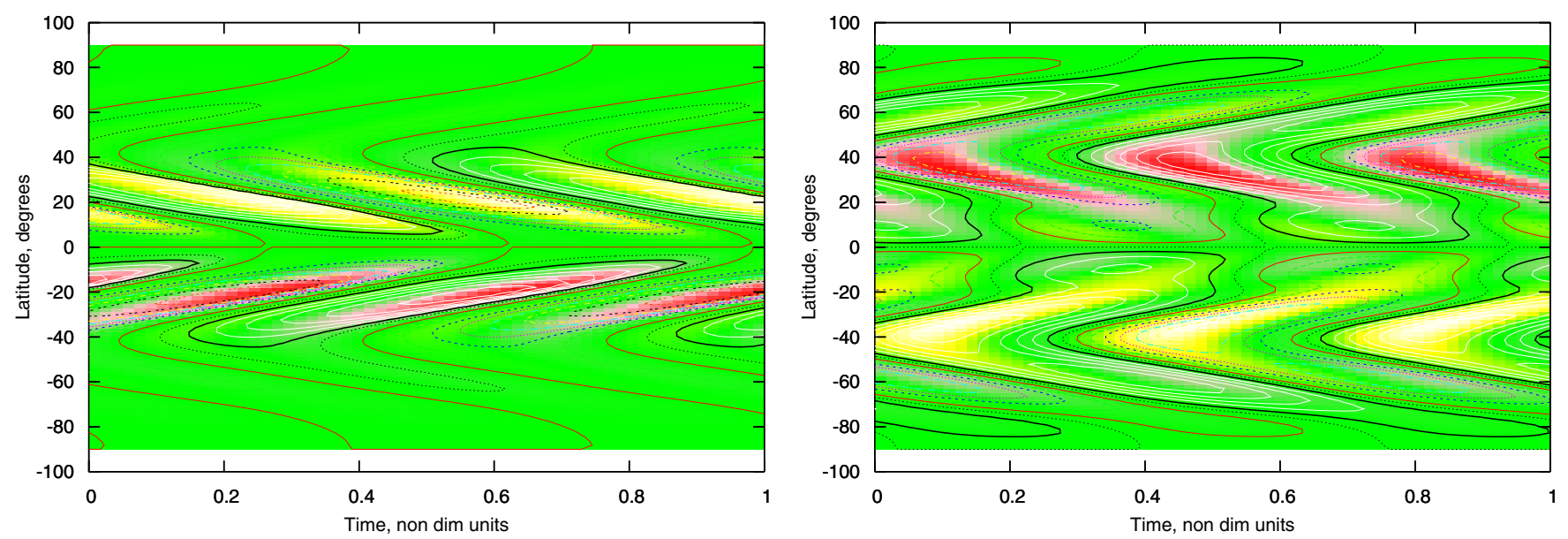

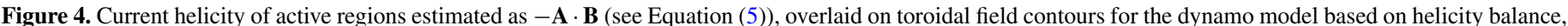

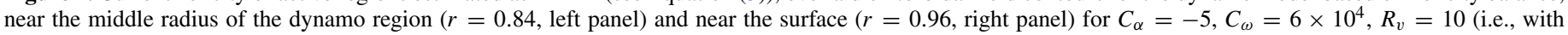
meridional circulation), and buoyancy parameter $\gamma=1$.

(A color version of this figure is available in the online journal.)

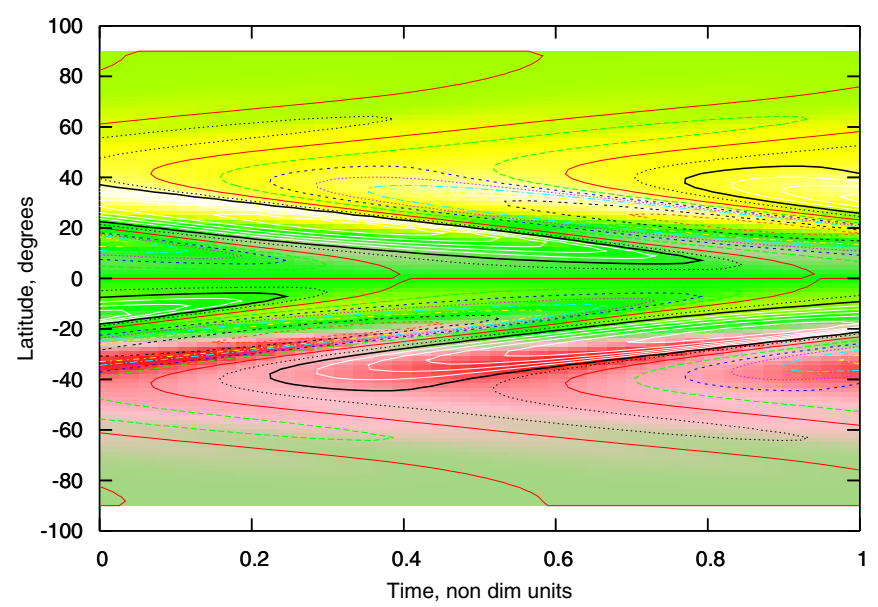

Figure 5. Small-scale current helicity $\chi_{c}$ overlaid on toroidal field contours for the dynamo model based on helicity balance, near the middle radius of the dynamo region. $C_{\alpha}=-5, C_{\omega}=6 \times 10^{4}, R_{v}=10$ (i.e., with meridional circulation), buoyancy parameter $\gamma=1$. The plots are for fractional radius 0.84 .

(A color version of this figure is available in the online journal.) concentrated in middle latitudes and helicity oscillations which are present in the model are almost invisible on the background of the intensive belt of constant helicity in middle latitudes. We doubt that such oscillations would be observable. We stress that, if this model produces any traveling helicity pattern, it is situated in the deep layers only. The pattern usually is much more similar to that presented in the right-hand panel of Figure 3 rather than to a traveling wave such as presented in Figure 2.

Taking our models as a whole, determination of further details of the location of the dynamo layer or the helicity sign inversion at some latitudes and phases of the solar cycle is beyond the scope of this investigation. Such a study would require a more complex simulation, which we hope to perform in the future.

The numerical models used here are extremely simple and cannot be expected to sample the full range of solutions that are accessible to a three-dimensional, highly stratified, extremely turbulent system with complex and unknown boundary conditions. In the present paper, we have only shown the theoretical possibility that the current helicity observed in solar active regions may trace the magnetic helicity of the large-scale dynamo generated field. We demonstrated that the results obtained with 
our simplified dynamo model are compatible with the observations. At the same time we note that such computationally complex and expensive simulations are still far from being able to reproduce the range of observed solar phenomena, and that for the immediate future mean field models will continue to play an important role. Indeed, they may provide more immediate physical insight.

The simple models which we have considered here were not intended to reproduce very fine details of the spatialtime distribution of helicity observable in the form of butterfly diagrams. We may again note that our modeling of helicity in the solar convective zone and active regions is still too simplified to be able to detect more detailed properties of the helicity dynamics.

While we feel that it is remarkable that there is a class of kinematic models that are able to reproduce the current helicity observations from vector magnetograms, we may again note that some contribution to the observed current helicity may be due to the surface effects caused by the differential rotation (Mackay \& Van Ballegooijen 2005; Yeates \& Mackay 2009) and not from the dynamo.

\section{CONCLUSIONS}

We conclude that current helicity of the magnetic field in active regions is a tracer of the magnetic helicity of the largescale magnetic field in the solar interior. We believe that this provides a unique option for tracing this quantity, which is very important for the solar dynamo. According to the observational data (Zhang et al. 2010), the current helicity in active regions is mainly negative in the northern hemisphere. Numerical models give a negative value for $-\mathbf{A} \cdot \mathbf{B}$ in the surface layer of the convective zone in the northern hemisphere.

Summarizing, we conclude that the current helicity of the magnetic field in active regions is expected to have the opposite sign to $\boldsymbol{A} \cdot \boldsymbol{B}$, evaluated at the depth at which the active region originates. Thus, the models presented here are consistent with the interpretation that the mechanism responsible for the sign of the observed helicity operates near the solar surface, cf. e.g., Kosovichev (2010). The mechanism of formation of the current helicity in active regions still requires further investigation.

We acknowledge the NORDITA dynamo programs of 2009 and 2011 for providing a stimulating scientific atmosphere. This collaborative work has been benefited from discussions at the Helicity Thinkshop meeting in Beijing in 2009. The work has been supported by Chinese grants NSFC 10733020, 10921303, 41174153, 11103037, NBRPC 2006CB806301, CAS KLCX2EW-T07, and joint NNSF-RBFR grant 10911120051. K.K., D.S., N.K., and D.M. acknowledge support from the National Astronomical Observatories of China during their visits, K.K. and D.S. are thankful to the joint project of NNSF of China and RFBR of Russia No. 08-02-92211, to RFBR grant 10-02-00960, to Visiting Professorship Programme 2009J2-12, as well as joint Chinese-Russian collaborative project from CAS.

\section{APPENDIX A}

\section{CURRENT HELICITY VERSUS THE MAGNETIC HELICITY}

Here, we relate the mean current helicity $\left\langle\mathbf{B}^{\mathrm{ar}} \cdot \operatorname{curl} \mathbf{B}^{\mathrm{ar}}\right\rangle$ with the magnetic helicity $\left\langle\mathbf{A}^{\mathrm{ar}} \cdot \mathbf{B}^{\mathrm{ar}}\right\rangle$. First we rewrite the mean current helicity from first principles with the use of permutation tensors as

$$
\begin{aligned}
& \left\langle\mathbf{B}^{\mathrm{ar}} \cdot \operatorname{curl} \mathbf{B}^{\mathrm{ar}}\right\rangle=\varepsilon_{m p q} \varepsilon_{m i j} \lim _{\mathbf{x} \rightarrow \mathbf{y}} \nabla_{p}^{\mathbf{x}} \nabla_{i}^{\mathbf{y}}\left\langle A_{q}^{\mathrm{ar}}(\mathbf{x}) B_{j}^{\mathrm{ar}}(\mathbf{y})\right\rangle \\
& =\lim _{\mathbf{x} \rightarrow \mathbf{y}}\left[\left(\nabla^{\mathbf{x}} \cdot \nabla^{\mathbf{y}}\right)\left\langle\mathbf{A}^{\mathrm{ar}}(\mathbf{x}) \cdot \mathbf{B}^{\mathrm{ar}}(\mathbf{y})\right\rangle-\nabla_{p}^{\mathbf{x}} \nabla_{q}^{\mathbf{y}}\left\langle A_{q}^{\mathrm{ar}}(\mathbf{x}) B_{p}^{\mathrm{ar}}(\mathbf{y})\right\rangle\right],
\end{aligned}
$$

where $\varepsilon_{i j n}$ is the fully antisymmetric Levi-Civita tensor, $R_{\odot}$ is the solar radius, $L_{\mathrm{ar}}$ is the spatial scale of an active region, and $\mathbf{r}=\mathbf{x}-\mathbf{y}$. The use of the full tensor notation and limits are needed here in order to separate the large-scale and small-scale variables and to obtain a simple final answer in scalar form when there is separation of scales. Since $\mathbf{r}=\mathbf{x}-\mathbf{y}$ is a small-scale variable, $\mathbf{R}=(\mathbf{x}+\mathbf{y}) / 2$ is a large-scale variable, the derivatives

$$
\begin{aligned}
& \nabla_{p}^{\mathbf{x}} \equiv \frac{\partial}{\partial x_{p}}=\frac{\partial}{\partial r_{p}}+\frac{1}{2} \frac{\partial}{\partial R_{p}}=-\nabla_{p}^{\mathbf{y}}+\frac{\partial}{\partial R_{p}}, \\
& \nabla_{p}^{\mathbf{y}} \equiv \frac{\partial}{\partial y_{p}}=-\frac{\partial}{\partial r_{p}}+\frac{1}{2} \frac{\partial}{\partial R_{p}}=-\nabla_{p}^{\mathbf{x}}+\frac{\partial}{\partial R_{p}} .
\end{aligned}
$$

This implies that

$$
\begin{aligned}
\nabla^{\mathbf{x}} \cdot \nabla^{\mathbf{y}} & =-\left(\frac{\partial^{2}}{\partial \mathbf{r}^{2}}-\frac{1}{4} \frac{\partial^{2}}{\partial \mathbf{R}^{2}}\right) \\
\nabla_{p}^{\mathbf{x}} \nabla_{q}^{\mathbf{y}} & =\nabla_{p}^{\mathbf{y}} \nabla_{q}^{\mathbf{x}}-\nabla_{p}^{\mathbf{y}} \frac{\partial}{\partial R_{q}}-\nabla_{q}^{\mathbf{x}} \frac{\partial}{\partial R_{p}}+\frac{\partial^{2}}{\partial R_{p} \partial R_{q}} .
\end{aligned}
$$

We take into account that $\operatorname{div} \mathbf{B}^{\mathrm{ar}}=0$ (i.e., $\nabla_{p}^{\mathbf{y}} B_{p}^{\mathrm{ar}}(\mathbf{y})=0$ ) and $\operatorname{div} \mathbf{A}^{\text {ar }}=0$ (i.e., $\nabla_{q}^{\mathbf{x}} A_{q}^{\text {ar }}(\mathbf{x})=0$ ). We also take into account that the characteristic scale of an active region is small compared with the thickness of the convection zone or the radius of the Sun. This implies that

$$
\nabla_{p}^{\mathbf{x}} \nabla_{q}^{\mathbf{y}}\left\langle A_{q}^{\mathrm{ar}}(\mathbf{x}) B_{p}^{\mathrm{ar}}(\mathbf{y})\right\rangle=\frac{\partial^{2}}{\partial R_{p} \partial R_{q}}\left\langle A_{q}^{\mathrm{ar}}(\mathbf{x}) B_{p}^{\mathrm{ar}}(\mathbf{y})\right\rangle \sim O\left(\frac{L_{\mathrm{ar}}^{2}}{R_{\odot}^{2}}\right),
$$

and therefore this term vanishes. This yields

$$
\left\langle\mathbf{B}^{\mathrm{ar}} \cdot \operatorname{curl} \mathbf{B}^{\mathrm{ar}}\right\rangle=-\left(\frac{\partial^{2}}{\partial r_{p} \partial r_{p}}\left\langle\mathbf{A}^{\mathrm{ar}} \cdot \mathbf{B}^{\mathrm{ar}}\right\rangle\right)_{\mathbf{r} \rightarrow 0}+O\left(\frac{L_{\mathrm{ar}}^{2}}{R_{\odot}^{2}}\right) .
$$

Now we take into account that the second derivative of the correlation function

$$
\left(\frac{\partial^{2}}{\partial r_{p} \partial r_{p}}\left\langle\mathbf{A}^{\mathrm{ar}} \cdot \mathbf{B}^{\mathrm{ar}}\right\rangle\right)_{\mathbf{r} \rightarrow 0}
$$

should be negative since as $\mathbf{r} \rightarrow 0$ the correlation function has a maximum. Thus, we finally obtain

$$
\left\langle\mathbf{B}^{\mathrm{ar}} \cdot \operatorname{curl} \mathbf{B}^{\mathrm{ar}}\right\rangle \approx \frac{1}{L_{\mathrm{ar}}^{2}}\left\langle\mathbf{A}^{\mathrm{ar}} \cdot \mathbf{B}^{\mathrm{ar}}\right\rangle+O\left(\frac{L_{\mathrm{ar}}^{2}}{R_{\odot}^{2}}\right) .
$$

Similar calculations relating the current helicity and the magnetic helicity in k-space can be found in Appendix C of Kleeorin \& Rogachevskii (1999).

\section{APPENDIX B}

\section{DETAILED DESCRIPTION OF THE DYNAMO MODEL}

The flux of the small-scale magnetic helicity is chosen in the form

$$
\begin{aligned}
\mathcal{F}= & \eta_{A}(B) B^{2}\left\{C_{1} \nabla\left[\chi^{v} \phi_{v}(B)\right]\right. \\
& \left.+C_{2} \chi^{v} \phi_{v}(B) \boldsymbol{\Lambda}_{\rho}\right\}-C_{3} \kappa \nabla \chi^{c}
\end{aligned}
$$


with $\Lambda_{\rho}=-\nabla \rho / \rho$ and $\kappa$ is the coefficient of turbulent diffusion of small-scale magnetic helicity (see below).

The quenching functions $\Phi_{v}(B)$ and $\Phi_{m}(B)$ appearing in the nonlinear $\alpha$ effect are given by

$$
\begin{aligned}
\Phi_{v}(B) & =\frac{1}{7}\left[4 \phi_{m}(B)+3 L(\sqrt{8} B)\right], \\
\Phi_{m}(B) & =\frac{3}{8 B^{2}}\left[1-\frac{\arctan (\sqrt{8} B)}{\sqrt{8} B}\right]
\end{aligned}
$$

(Rogachevskii \& Kleeorin 2000), where $L(y)=1-2 y^{2}+$ $2 y^{4} \ln \left(1+y^{-2}\right)$.

The nonlinear turbulent magnetic diffusion coefficients for the mean poloidal and toroidal magnetic fields, $\eta_{A}(B)$ and $\eta_{B}(B)$, are given in dimensionless form by

$$
\begin{gathered}
\eta_{A}(B)=A_{1}(4 B)+A_{2}(4 B), \\
\eta_{B}(B)=A_{1}(4 B)+\frac{3}{2}\left[2 A_{2}(4 B)-A_{3}(4 B)\right],
\end{gathered}
$$

(Rogachevskii \& Kleeorin 2004). For the case of weak magnetic field the turbulent diffusion coefficients are (in units of the reference value $\eta_{T 0}$ )

$$
\eta_{A}=1-\frac{96}{5} B^{2}, \quad \eta_{B}=1-32 B^{2}
$$

while for strong magnetic fields the scaling is

$$
\eta_{A}=\frac{1}{8 B^{2}}, \quad \eta_{B}=\frac{1}{3 \sqrt{2} B} .
$$

The transition from one asymptotic form to the other can be thought of as occurring in the vicinity of $B \sim B_{\mathrm{eq}} / 4$.

The nonlinear drift velocities of poloidal and toroidal mean magnetic fields, $\boldsymbol{V}^{A}(B)$ and $\boldsymbol{V}^{B}(B)$, are given in dimensionless form by

$$
\begin{aligned}
& \boldsymbol{V}^{A}(B)=V_{1}(B) \frac{\boldsymbol{\Lambda}_{B}}{2}+\frac{V_{2}(B)}{r}\left(\boldsymbol{e}_{r}+\cot \theta \boldsymbol{e}_{\theta}\right)+\boldsymbol{V}_{\rho}(B), \\
& \boldsymbol{V}^{B}(B)=\frac{V_{3}(B)}{r}\left(\boldsymbol{e}_{r}+\cot \theta \boldsymbol{e}_{\theta}\right)+\boldsymbol{V}_{\rho}(B),
\end{aligned}
$$

where

$$
\begin{aligned}
V_{1}(B) & =\frac{3}{2} A_{3}(4 B)-2 A_{2}(4 B), \\
V_{2}(B) & =\frac{1}{2} A_{2}(4 B), \\
V_{3}(B) & =\frac{3}{2}\left[A_{2}(4 B)-A_{3}(4 B)\right], \\
V_{\rho}(B) & =\frac{1}{2} \Lambda_{\rho}\left[-5 A_{2}(4 B)+\frac{3}{2} A_{3}(4 B)\right] .
\end{aligned}
$$

The asymptotic formulae for these velocities are given by

$$
\begin{aligned}
\boldsymbol{V}^{A} & =\frac{32}{5} B^{2}\left[\boldsymbol{\Lambda}_{B}+3 \boldsymbol{\Lambda}_{\rho}-\frac{\boldsymbol{e}_{r}+\cot \theta \boldsymbol{e}_{\theta}}{r}\right], \\
\boldsymbol{V}^{B} & =\frac{32}{5} B^{2}\left[3 \boldsymbol{\Lambda}_{\rho}-\frac{\boldsymbol{e}_{r}+\cot \theta \boldsymbol{e}_{\theta}}{r}\right]
\end{aligned}
$$

for a weak magnetic field, and

$$
\begin{aligned}
\boldsymbol{V}^{A} & =-\frac{1}{3 \sqrt{8} B}\left[\boldsymbol{\Lambda}_{B}+2 \frac{\boldsymbol{e}_{r}+\cot \theta \boldsymbol{e}_{\theta}}{r}\right]+\frac{5}{16 B^{2}} \boldsymbol{\Lambda}_{\rho}, \\
\boldsymbol{V}^{B} & =\frac{4}{3 \sqrt{8} B} \frac{\boldsymbol{e}_{r}+\cot \theta \boldsymbol{e}_{\theta}}{r}+\frac{5}{16 B^{2}} \boldsymbol{\Lambda}_{\rho}
\end{aligned}
$$

for strong fields. Here $\Lambda_{B}=\left(\nabla \boldsymbol{B}^{2}\right) / \boldsymbol{B}^{2}, \boldsymbol{e}_{r}$ and $\boldsymbol{e}_{\theta}$ are unit vectors in the $r$ and $\theta$ directions of spherical polar coordinates, $\left[\boldsymbol{\Lambda}_{\rho}\right]_{r}=-d \ln \rho / d r$, and $\left[\boldsymbol{\Lambda}_{B}\right]_{r}=d \ln B^{2} / d r$. See, for details, Rogachevskii \& Kleeorin (2004, Equations (18), (19), (22)-(24)), which have been rewritten here in spherical geometry.

The functions $A_{k}(y)$ are

$$
\begin{aligned}
& A_{1}(y)=\frac{6}{5}\left[\frac{\arctan y}{y}\left(1+\frac{5}{7 y^{2}}\right)+\frac{1}{14} L(y)-\frac{5}{7 y^{2}}\right], \\
& A_{2}(y)=-\frac{6}{5}\left[\frac{\arctan y}{y}\left(1+\frac{15}{7 y^{2}}\right)-\frac{2}{7} L(y)-\frac{15}{7 y^{2}}\right], \\
& A_{3}(y)=-\frac{2}{y^{2}}\left[\frac{\arctan y}{y}\left(y^{2}+3\right)-3\right] .
\end{aligned}
$$

The nonlinear quenching of the turbulent magnetic diffusion of the magnetic helicity is given by

$$
\kappa(B)=\frac{1}{2}\left[1+A_{1}(4 B)+\frac{1}{2} A_{2}(4 B)\right] .
$$

The coefficient of turbulent diffusion of magnetic helicity $\kappa$ also has a dependence on $B$, namely, $\kappa(B)=1-24 B^{2} / 5$ for a weak magnetic field and

$$
\kappa(B)=\frac{1}{2}\left(1+\frac{3 \pi}{40 B}\right)
$$

for the strong field limit.

\section{REFERENCES}

Baker, N., \& Temesvary, S. 1966, Table of Convective Stellar Envelope Models (New York: Goddaid Inst.), 312

Baliunas, S., Frick, P., Moss, D., et al. 2006, MNRAS, 365, 181

Bao, S. D., \& Zhang, H. Q. 1998, ApJ, 496, L43

Blackman, E. G., \& Brandenburg, A. 2003, ApJ, 584, L99

Blackman, E. G., \& Field, G. B. 2000a, ApJ, 534, 984

Blackman, E. G., \& Field, G. B. 2000b, MNRAS, 318, 724

Brandenburg, A. 2005, ApJ, 625, 539

Brandenburg, A. 2007, Highlights Astron., 14, 291

Brandenburg, A., Kemel, K., Kleeorin, N., Mitra, D., \& Rogachevskii, I. 2011, ApJ, 740, L50

Brandenburg, A., Kleeorin, N., \& Rogachevskii, I. 2010, Astron. Nachr., 331, 5

Brandenburg, A., \& Subramanian, K. 2005, Phys. Rep., 417, 1

Cally, P. S., Dikpati, M., \& Gilman, P. A. 2003, ApJ, 582, 1190

Choudhuri, A. R., Chatterjee, P., \& Nandy, D. 2004, ApJ, 615, L57

Dikpati, M., \& Gilman, P. A. 2001, ApJ, 559, 420

Gilman, P. A., \& Dikpati, M. 2000, ApJ, 528, 552

Hagino, M., \& Sakurai, T. 2004, PASJ, 56, 831

Kleeorin, N., Kuzanyan, K., Moss, D., et al. 2003, A\&A, 409, 1097

Kleeorin, N., Mond, M., \& Rogachevskii, I. 1996, A\&A, 307, 293

Kleeorin, N., Moss, D., Rogachevskii, I., \& Sokoloff, D. 2000, A\&A, 361, L5

Kleeorin, N., \& Rogachevskii, I. 1999, Phys. Rev. E, 59, 6724

Kleeorin, N., Rogachevskii, I., \& Ruzmaikin, A. 1995, A\&A, 297, 159

Kosovichev, A. G. 2010, Sol. Phys., in press (arXiv:1010.4927)

Krause, F., \& Rädler, K.-H. 1980, Mean-field Magnetohydrodynamics and Dynamo Theory (Oxford: Pergamon)

Mackay, D. H., \& Van Ballegooijen, A. A. 2005, ApJ, 621, L77 
Mitra, D., Moss, D., Tavakol, R., \& Brandenburg, A. 2011, A\&A, 526, 138 Moffatt, H. K. 1978, Magnetic Field Generation in Electrically Conducting Fluids (Cambridge: Cambridge Univ. Press)

Moss, D., \& Brooke, J. 2000, MNRAS, 315, 521

Moss, D., Shukurov, A., \& Sokoloff, D. 1999, A\&A, 343, 120

Moss, D., Sokoloff, D., \& Lanza, A. F. 2011, A\&A, 531, 43

Moss, D., Tuominen, I., \& Brandenburg, A. 1990, A\&A, 228, 284

Parfrey, K. P., \& Menou, K. 2007, ApJ, 667, L207

Parker, E. 1955, ApJ, 122, 293

Pevtsov, A. A., Canfield, R. C., \& Metcalf, T. R. 1994, ApJ, 425, L117

Pouquet, A., Frisch, U., \& Leorat, J. 1976, J. Fluid Mech, 77, 321

Rogachevskii, I., \& Kleeorin, N. 2000, Phys. Rev. E, 61, 5202
Rogachevskii, I., \& Kleeorin, N. 2004, Phys. Rev. E, 70, 046310

Rogachevskii, I., \& Kleeorin, N. 2007, Phys. Rev. E, 76, 056307

Seehafer, N. 1990, Sol. Phys., 125, 219

Shukurov, A., Sokoloff, D., Subramanian, K., \& Brandenburg, A. 2006, A\&A, 448, L33

Spruit, H. C. 1974, Sol. Phys., 34, 277

Xu, H., Gao, Y., Popova, H. P., et al. 2009, Astron. Rep., 53, 160

Yeates, A. R., \& Mackay, D. H. 2009, Sol. Phys., 254, 77

Zeldovich, Ya. B., Ruzmaikin, A. A., \& Sokoloff, D. D. 1983, Magnetic Fields in Astrophysics (New York: Gordon and Breach)

Zhang, H., Sakurai, T., Pevtsov, A., et al. 2010, MNRAS, 402, L30

Zhang, H., Sokoloff, D., Rogachevskii, I., et al. 2006, MNRAS, 365, 276 